\title{
Validación de un Paradigma de Razonamiento Abstracto Para Resonancia Magnética Funcional (RMf)
}

\author{
Validation Of An Abstract Reasoning Paradigm \\ For Functional Magnetic Resonance (fMR)
}

Fernando Shunta Cocha, Fernando Estévez Abad, Daniel Aguirre Reyes, Omar Alvarado Cando, Andrés López Hidalgo, Pablo Peña Tapia, Alexandra Bueno Pacheco

\section{Resumen}

Los paradigmas son las tareas experimentales que se usan para mapear el cerebro a través de Resonancia Magnética funcional. Su validación previa es fundamental para garantizar un registro exitoso de la actividad cognitiva a investigar. Se validó un paradigma de razonamiento abstracto formado por una tarea de analogías semánticas y una tarea de analogías visuales en jóvenes-adultos entre 18 y 30 años de edad de la ciudad de Cuenca-Ecuador. Se programó el paradigma en el software libre PsychoPy 3 para experimentos de neurociencia. Los resultados indicaron que el paradigma fue comprensible y sencillo de resolver. También se halló que el tiempo promedio de respuesta en la tarea de razonamiento semántico fue significativamente menor al tiempo promedio de resolución de la tarea de razonamiento visual. Por lo tanto, se pudo establecer el número de estímulos necesarios y su tiempo promedio de exposición para garantizar la potencia de contraste y significancia estadística al utilizarlo en un Resonador Magnético.

Palabras clave: paradigma, validación, resonancia magnética funcional, razonamiento abstracto, PsychoPy.

\section{Abstract}

The paradigms are the experimental tasks that are used to map the brain through functional Magnetic Resonance Imaging. Its previous validation is essential to guarantee a successful record of the cognitive activity. A paradigm of abstract reasoning formed by a task of semantic and visual analogies was validated in young-adults between 18 and 30 years in the city of CuencaEcuador. The paradigm was programmed in PsychoPy 3, a free software for neuroscience experiments. The results indicated that the experimental paradigm was understandable and easy to solve. It was also found that the average response time in the semantic reasoning task was significantly lower than the average resolution time of the visual reasoning task. Therefore, it was possible to establish the number of stimuli and their average exposure time needed for a functional Magnetic Resonance Imaging.

Keywords: paradigm, validation, functional magnetic resonance imaging, abstract reasoning, PsychoPy.

Rev. Ecuat. Neurol. Vol. 29, N² 2020

\section{Introducción}

Se calcula que a nivel mundial se publican diariamente tres estudios que emplean Resonancia Magnética funcional (RMf). En el Ecuador, la RMf aún está subutilizada en el campo de la investigación, puesto que su rol se ha centrado en el mapeo cerebral antes y después de una intervención neuroquirúrgica. No obstante, existen trabajos pioneros desarrollados en la ciudad de Loja que han empleado RMf para mapear procesos cognitivos superiores como las funciones ejecutivas, la atención, el lenguaje, entre otros--6

El razonamiento abstracto es una característica distintiva de la inteligencia humana, forma parte de las funciones ejecutivas y se ha localizado en las áreas 9 y 46 de Brodmann de la corteza prefrontal dorsolateral? La abstracción es un nivel elevado del pensamiento y se asocia a las capacidades de deducir, hipotetizar, sintetizar, interpretar y analizar fenómenos de forma simbólica, separando las cualidades de un objeto o fenómeno para captar su significado o esencia.

Es decir, prescinde de estímulos concretos y genera conclusiones a partir de diversas operaciones intelectuales, tales como el razonamiento analógico, que es una forma de razonamiento relacional que depende de la capacidad para considerar y comparar relaciones e integrar y unir esas relaciones. La identificación de similitudes entre objetos, o situaciones 
aparentemente diferentes, permite unir varios dominios de conocimiento y transferir soluciones de un dominio a otro.

En el razonamiento analógico las similitudes son principalmente relacionales, puesto que se centran en las relaciones entre los componentes de los objetos, más que en los objetos en sí mismos? Por tanto, el razonamiento relacional apoya al pensamiento abstracto que, por lo general, se evalúa a través de pruebas de papel y lápiz que incluyen ejercicios de exclusión, analogías, silogismos, tareas de secuenciación, entre otras.

Sin embargo, el razonamiento abstracto también se ha mapeado a través de varias técnicas de neuroimagen como la RMf,-12 la cual genera imágenes del funcionamiento en vivo del cerebro y elabora mapas visuales de localización topográfica de la actividad cognitiva a través de la aplicación de paradigmas, los cuales conforman la tarea experimental a realizarse dentro de un Resonador Magnético (RM). El objetivo de un paradigma es activar procesos mentales específicos para identificar la arquitectura cognitivo-funcional subyacente a ellos. ${ }^{13,14}$

El diseño de paradigmas más empleado en la investigación con RMf se conoce como diseño en bloques, que consiste en ejercicios que alternan una condición experimental ("activación") y una control ("línea base" o "reposo"). ${ }^{15} \mathrm{En}$ reposo el sujeto no realiza ninguna acción o se presentan estímulos ajenos a la tarea principal, mientras que en actividad el participante resuelve el paradigma propuesto. Las ventajas del diseño de bloques son su sencillez de aplicación y su alta potencia estadística. ${ }^{15}$ Todo experimento con RMf debe comparar, al menos, dos condiciones (experimental y control) de tal forma que sea posible estudiar las diferencias en la señal asociadas a cada una de ellas. ${ }^{14}$

Las características a considerar en un paradigma de diseño de bloques son su número y duración, el tiempo de exposición de los estímulos y la cantidad de condiciones. A mayor número de bloques, mayor potencia de contraste y de significación estadística. La duración óptima de bloques se encuentra entre los 14 segundos (s) y 20 s, aunque también se pueden usar bloques más largos (de $30 \mathrm{~s}$ ) dependiendo de la potencia estadística que se espere obtener del experimento. ${ }^{14}$ No obstante, se debe cuidar el tiempo de duración del experimento ya que se pueden generar factores negativos como la ansiedad o cansancio disminuyendo la validez psicológica del estudio. Así, para aumentar la sensibilidad del estudio se debe maximizar el tiempo en el que el estímulo esté presente y minimizar el intervalo entre estímulos.

La duración de un paradigma también depende de la potencia del RM, que se mide en unidades Tesla $(\mathrm{T}){ }^{16}$ Por ejemplo, para que un RM de $1.5 \mathrm{~T}$ obtenga la misma cantidad de señal percibida por las antenas de un resonador de $3 \mathrm{~T}$, el paradigma debe durar cuatro veces más. Es decir, un tiempo aproximado de cuatro minutos alternados entre períodos de reposo y actividad. ${ }^{17}$ Por tanto, antes de emplear cualquier paradigma en un RM, éste debe ser validado en sujetos sanos para su aplicación. ${ }^{18}$
Los paradigmas más frecuentes en la aplicación clínica con RMf por su utilidad, estandarización, confiabilidad y validez se han centrado en el estudio de procesos motores y de lenguaje. ${ }^{14}$ Sin embargo, también existen trabajos que exploran otras funciones cognitivas como el razonamiento abstracto. En ese sentido, un meta-análisis de literatura de técnicas de neuroimagen para mapear el razonamiento abstracto mostró que la mayoría de estudios revisados emplearon paradigmas con ejercicios de términos analógicos y tareas similares a las matrices progresivas de Raven?

Por su parte, Whitaker et al.$^{10}$ diseñaron dos paradigmas basados en la prueba breve de inteligencia K-Bit 2 para analizar los patrones de activación neurológica mediante RMf en niños y adolescentes. Los paradigmas incluían ejercicios de analogías proporcionales y analogías de coincidencias semánticas formados por imágenes y palabras a color. El tiempo de exposición de cada estímulo fue de $10 \mathrm{~s}$, en los que se mapeó la elección de respuesta y el establecimiento de relaciones perceptuales abstractas entre imágenes.

Modi et al. ${ }^{11}$ emplearon un paradigma de razonamiento abstracto visuoespacial en un RM para evaluar la relación entre ansiedad y la eficiencia de procesamiento mental en sujetos sanos. Los estímulos estaban formados por figuras geométricas secuenciales en aumento progresivo con un tiempo de exposición de 4s. En la fase de actividad se presentaron dos opciones de respuesta que el sujeto seleccionaba para completar la serie de estímulos previamente presentados.

Hammer et al..$^{12}$ utilizaron un paradigma para RMf con el fin de investigar si las diferencias individuales en razonamiento analógico se pueden explicar por la actividad de algunas regiones cerebrales prefrontales o, si en ella, está involucrado un patrón de actividad distribuido en una red neurológica más amplia. El paradigma consistió en estímulos que incluían, al mismo tiempo, imágenes y palabras. El tiempo de exposición de cada estímulo fue de $8 \mathrm{~s}$ en los que el sujeto determinaba si la similitud relacional entre los pares de imágenes y las palabras diana eran válidas.

En Ecuador, Ramos et al. ${ }^{19}$ adaptaron y validaron tres tareas experimentales (SIMON, Go/No-Go y Stroop) usando un programa informático de acceso libre conocido como "Psychology Experiment Building Language" (PEBL). Programaron las tareas en el PEBL usando imágenes y palabras a color, realizaron una aplicación piloto con el fin de mejorar las tareas y, por último, aplicaron las versiones finales a 100 estudiantes entre 6 y 15 años de edad. Si bien la validación no se realizó para su aplicación en un RM, esto muestra el potencial de uso de los programas informáticos gratuitos en nuestro medio y la capacidad de adaptación de las tareas a un RMf.

Como puede observase, los paradigmas empleados en los estudios descritos son diversos en cuanto al tiempo de exposición, tipos de estímulos y uso de programas informáticos. Unos se basan en pruebas psicológicas o neuropsicológicas y otros en ejercicios modificados pertenecientes a otros autores. 
Es, por tanto, un desafío para el neuropsicólogo la creación de paradigmas validados para RM que mapeen procesos cognitivos superiores que aporten nuevos conocimientos a las neurociencias. En ese sentido, en el Ecuador contamos sólo con cinco estudios relacionados con paradigmas y $\mathrm{RMf}^{2-6}$ pero no existen antecedentes de validaciones previas.

De esta manera, el presente estudio fue pionero en este país y aportó las bases metodológicas para futuras investigaciones que resulten en la creación de nuevos paradigmas válidos y confiables para técnicas de neuroimagen. Así, el objetivo de nuestra investigación fue diseñar y validar estadísticamente un paradigma de razonamiento abstracto para RMf compuesto por una tarea de razonamiento semántico y una tarea de razonamiento visual.

\section{Metodología \\ Diseño metodológico}

Se realizó una investigación cuantitativa de alcance explorativo ya que se trató de un tema que no se ha abordado antes. El diseño de investigación fue descriptivotranseccional puesto que los datos se recolectaron en un momento dado.

\section{Hipótesis de investigación}

Hi: "El tiempo de respuesta en la tarea de razonamiento visual será mayor al tiempo de respuesta en la tarea de razonamiento semántico"

Ho: "El tiempo de respuesta en la tarea de razonamiento visual no será mayor al tiempo de respuesta en la tarea de razonamiento semántico"

\section{Contexto, población y muestra}

La investigación se realizó en la ciudad de CuencaEcuador con 18 estudiantes universitarios de la carrera de medicina entre 18 y 30 años de edad. No hubo grupo control. La muestra fue seleccionada por conveniencia debido a la facilidad de acceso y disponibilidad de tiempo de los participantes. Se recogieron los datos demográficos de los sujetos y se les asignó un código de registro para mantener su anonimato. Asimismo, se les comunicó el objetivo del estudio y firmaron el consentimiento informado, conscientes de que su participación en la investigación no tenía algún impacto negativo en su salud o remuneración económica.

\section{Procedimiento}

Se diseñaron varios paradigmas de razonamiento abstracto hasta obtener una versión final que fue aprobada por un comité de expertos formado por una neuropsicóloga, un magister en neurociencias y un ingeniero de procesamiento de imágenes médicas. El paradigma final incluyó una tarea de razonamiento semántico y una tarea de razonamiento visual (Figura 1 a y b).

Posteriormente, se adaptó el paradigma al software de acceso libre para experimentos de neurociencias PsychoPy $3,{ }^{21}$ en el cual se programaron los períodos de reposo y actividad, la secuencia de presentación de los estímulos, la selección de teclas de respuesta y la organización de los datos relacionados con el tipo de respuesta y su tiempo de emisión por cada sujeto. El paradigma fue administrado en un computador portátil de 15 pulgadas, con un procesador Intel Core i5 de 2,5 GHz.

Se inició con la aplicación a papel y lápiz de un reactivo muestra por cada tarea del paradigma. Luego, se corrió el experimento en el computador, indicando a los sujetos que trabajasen lo más rápido posible. El programa tiene la capacidad de recopilar el tiempo de respuesta en minutos, segundos y milisegundos.

Finalmente, se obtuvieron las respuestas de los sujetos en una hoja de cálculo que se ordenaron en una base de datos para su procesamiento estadístico.

\section{Instrumentos}

A). Paradigma de razonamiento abstracto

Se creó un paradigma de razonamiento abstracto compuesto por dos tareas, una de analogías semánticas y otra de analogías visuales basadas en las subpruebas de "semejanzas" y "conceptos con dibujos" de la Escala de Inteligencia de Wechsler para niños versión IV.22
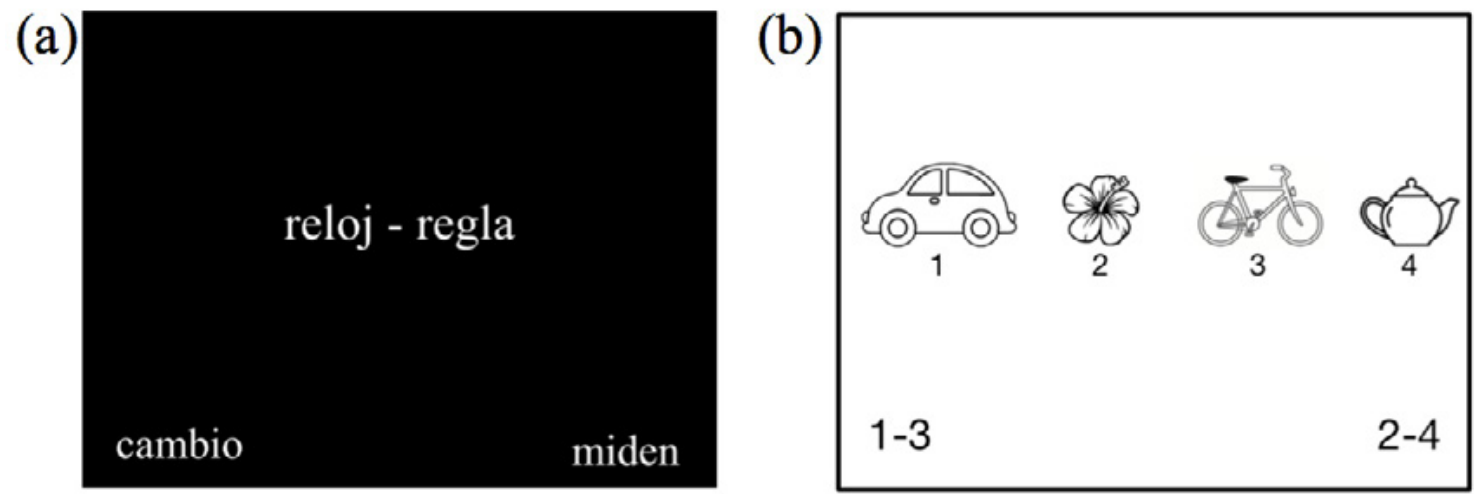

Figura 1 a y b. Ejemplos de estímulos de tarea de analogías semántucas y visuales. 
La tarea de analogías semánticas consistió en mostrar en el centro del monitor dos palabras relacionadas analógicamente entre sí (Figura 1a). El sujeto debía elegir su respuesta entre dos opciones colocadas en la parte inferior izquierda y derecha de la pantalla apretando la letra " $q$ " (izquierda),y la letra "p" (derecha). Se seleccionaron esas letras porque se encuentran paralelas a las opciones de respuestas presentadas en el monitor. Con ello, se minimizó el "efecto Simon" relacionado con el tiempo de reacción de acuerdo a la fuente del estímulo. 23,24 De las opciones de respuesta, una era correcta y otra incorrecta. Cuando el sujeto seleccionaba una opción, pasaba automáticamente el siguiente estímulo.

Esta tarea estuvo compuesta por diez reactivos presentados en dos bloques de cinco cada uno, separados por un período de reposo de $5 \mathrm{~s}$. El tiempo de exposición de cada estímulo fue indefinido, por lo que la emisión de respuesta dependió de la velocidad de cada participante. Así, se registró el tiempo exacto de respuesta por cada reactivo.

La tarea de analogías visuales consistió en mostrar cuatro imágenes enumeradas a blanco y negro y de trazos sim-

Figura 2. Secuencia del paradigma y orden de presentación de tiempos de reposo y actividad de las tareas de razonamiento analógico semántico y visual.

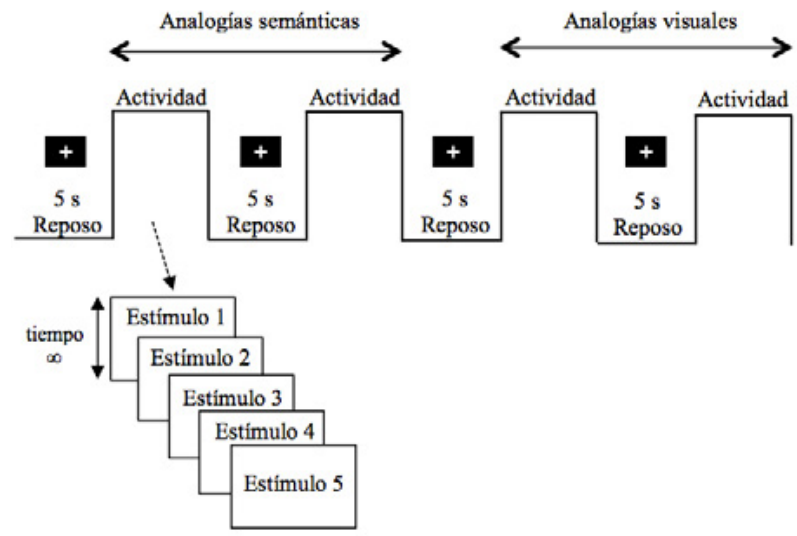

ples colocadas horizontalmente en el centro del monitor sobre un fondo blanco (Figura 1b). Se eligió ese diseño gráfico para minimizar, en una futura aplicación en RMf, la activación de otras áreas cerebrales relacionadas con elementos como el color, expresiones, entre otras. De las cuatro imágenes presentadas, sólo dos se relacionaban analógicamente entre sí, mientras que las restantes eran distractores. Se eligieron dos categorías abstractas para los estímulos: medios de transporte y útiles escolares y de oficina. Las imágenes se obtuvieron de la página de acceso libre dibujos.net.

Las opciones de respuesta, una correcta y otra incorrecta, estaban expresadas en pares de números colocados en la parte inferior izquierda y derecha del monitor. El número de estímulos y su presentación siguió la misma metodología de la tarea de razonamiento semántica.

El paradigma se presentó siguiendo un diseño de bloques para RMf (Figura 2): en reposo se presentó una cruz blanca sobre un fondo negro durante $5 \mathrm{~s}$. Transcurrido ese período continuaban los bloques de actividad con un tiempo indefinido de exposición de cada estímulo, de tal manera que la emisión de respuesta dependió de la velocidad de cada sujeto.

\section{B). Programa PsychoPy, versión 3}

Es un programa libre creado en el 2007 por Jonathan Peirce que usa lenguaje Python para crear una gran variedad de estímulos visuales y auditivos en computadoras personales que le permiten interactuar con equipos externos para su uso en RMf, Electroencefalograma (EEG), Magnetoencefalografía (MEG), entre otras. ${ }^{21}$ El paradigma se programó en PsychoPy tal y como se muestra en la Figura 3.

\section{Análisis de datos}

El procesamiento estadístico de los datos demográficos de la muestra se realizó mediante pruebas de estadística descriptiva para calcular medidas de tendencia central, dispersión y distribución de frecuencias y porcentajes.

Figura 3. Progamación del paradigma en PsychoPy3.

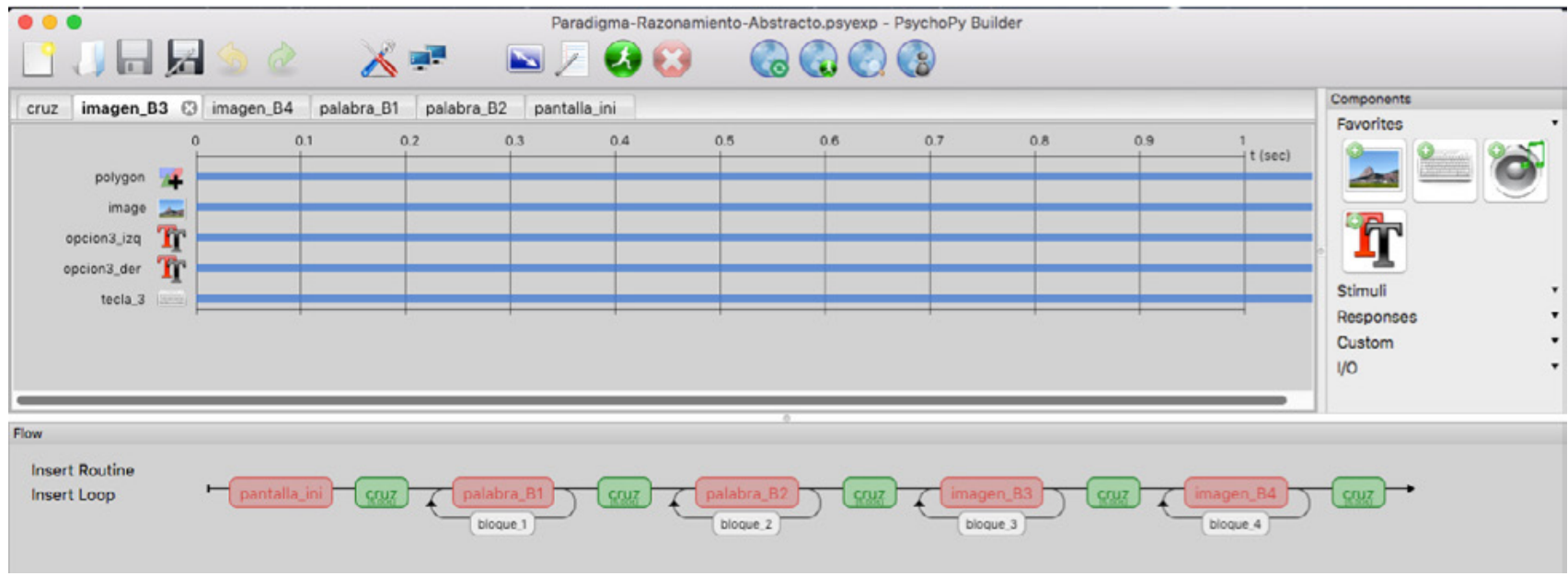


Tabla 1. Características demográficas de la muestra.

\begin{tabular}{|c|c|c|c|c|c|c|}
\hline Variable & Media + DE & Mediana & Rango & & $N(18)$ & $\%$ \\
\hline \multirow[t]{4}{*}{ Sexo } & - & - & - & Masculino & 12 & 66.66 \\
\hline & - & - & - & Femenino & 6 & 33.33 \\
\hline & & & & $19-20$ & 2 & 11.11 \\
\hline & & & & $21-22$ & 7 & 38.88 \\
\hline \multirow[t]{3}{*}{ Edad } & $22.5+2.15$ & 22 & $19-28$ & $23-24$ & 7 & 38.88 \\
\hline & & & & $25-26$ & 1 & 5.55 \\
\hline & & & & $27-28$ & 1 & 5.55 \\
\hline \multirow[t]{2}{*}{ Escolaridad (años) } & $14.89+1.02$ & 15 & $13-16$ & $<14$ años & 8 & 44.44 \\
\hline & & & & $>14$ años & 10 & 55.55 \\
\hline
\end{tabular}

En el análisis estadístico del paradigma se empleó la prueba $t$ (de Student). El procesamiento de datos se realizó en Microsoft Excel 2011 para MacOS versión 14.0.0 y el programa R-studio para MacOS versión 1.1.463.

\section{Resultados}

La siguiente tabla detalla las características de los participantes:

Como se ve en la Tabla 1, la muestra estuvo conformada por jóvenes-adultos entre 19 y 28 años de edad. De ellos, el 66\% fueron hombres y el 33\% fueron mujeres. El promedio de edad fue de 22 años de edad y el promedio de escolaridad fue de 14 años de estudio.

a.

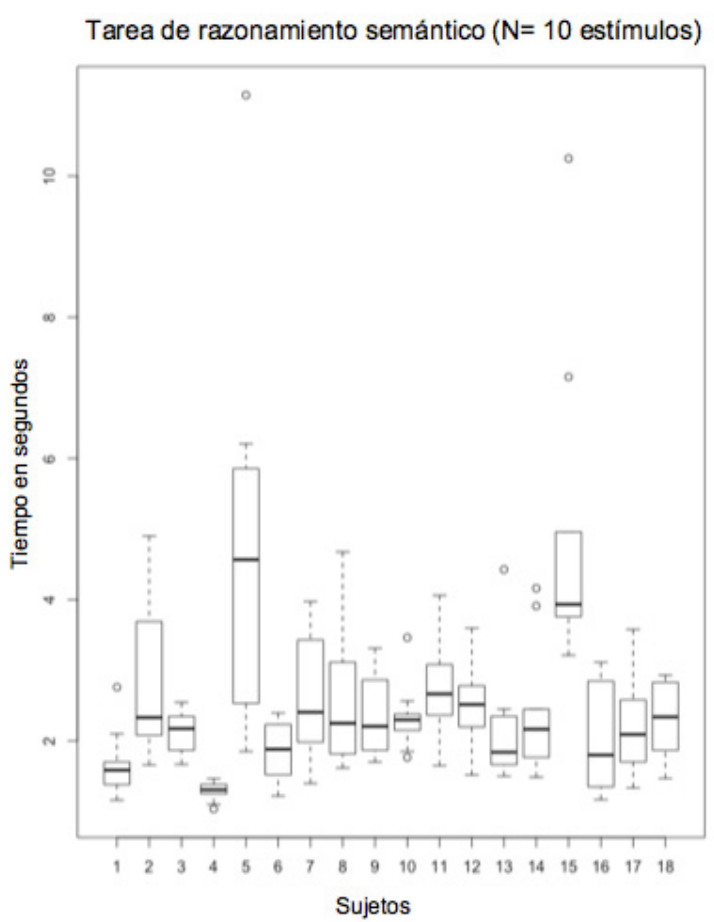

Previo al análisis estadístico del paradigma, se realizó un boxplot del tiempo de respuesta de los estímulos de las dos tareas del paradigma con el fin de identificar datos atípicos a un nivel de confianza del 95\% (Figuras 4a-b).

Como se observa en la Figura 4 el sujeto número 15 presentó datos atípicos fuera del nivel de confiabilidad en el tiempo de respuesta en las dos tareas del paradigma, por lo que se lo eliminó de la muestra inicial, que se redujo de 18 a 17 participantes.

A continuación, se realizó la prueba estadística "Shapiro-Wilk" para verificar si los datos del tiempo promedio de respuesta de los 17 sujetos en cada estímulo de

b.

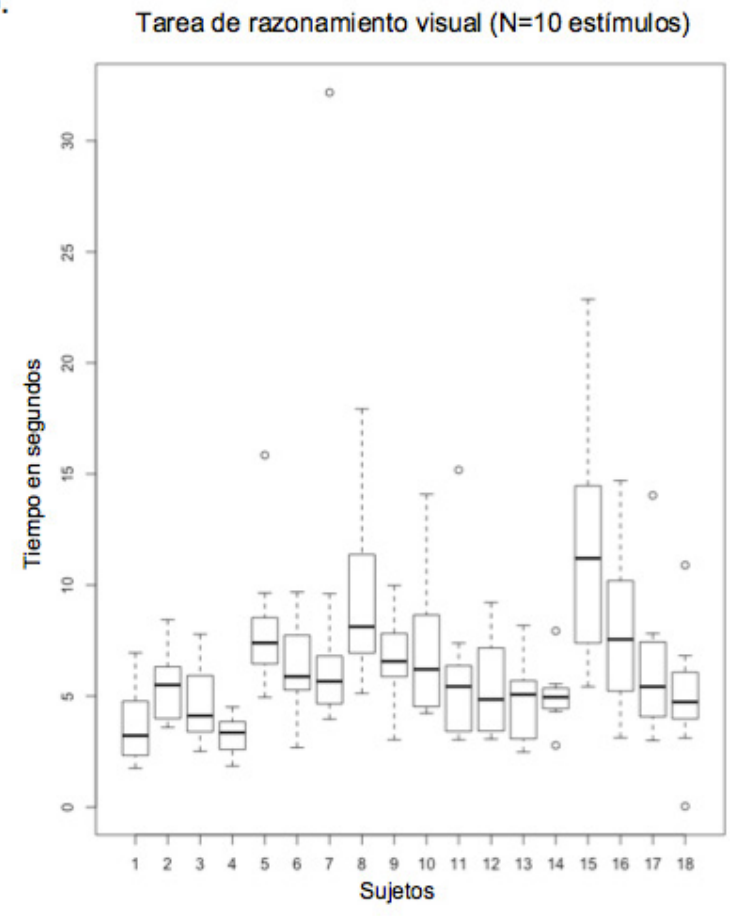

Figura 4. Boxplot del tiempo directo de respuesta generados por los 18 sujetos de los diez estímulos de la tarea de razonamiento semántico (a) y visual (b) 
Tabla 2. Resultados de la prueba Shapiro-Wilk del tiempo promedio de respuesta de los 10 estímulos de cada tarea del paradigma.

\begin{tabular}{|lccc|}
\hline Tarea & W & p-valor & $\begin{array}{c}\text { Nivel de significancia } \\
(\mathbf{a}=\mathbf{0 . 0 5})\end{array}$ \\
\hline Semántico (10 estímulos) & 0.929 & 0.44 & $\begin{array}{c}\text { Datos con } \\
\text { distribución normal }\end{array}$ \\
\hline Visual (10 estímulos) & 0.9591 & 0.77 & $\begin{array}{c}\text { Datos con } \\
\text { distribución normal }\end{array}$ \\
\hline
\end{tabular}

la tarea de razonamiento semántico y razonamiento visual estaban distribuidos normalmente (Tabla 2).

La Tabla 2 muestra que el p-valor en las dos tareas del paradigma es mayor al nivel de significancia de 0.05 , por lo que, con un $95 \%$ de confiabilidad, los datos del tiempo promedio de respuesta en cada estímulo se distribuyeron normalmente.

Con estos resultados, se pasó a realizar el análisis estadístico del paradigma. A continuación se muestran las frecuencias absolutas del número de aciertos generados en los 20 estímulos del paradigma (Figura 5).

En la Figura 5 se aprecia que 17 aciertos se presentaron 14 veces $(70 \%), 16$ aciertos se repitieron en 3 ocasiones (15\%). Por tanto, el $85 \%$ de los participantes presentaron respuestas correctas en el paradigma.

Posteriormente, se empleó la prueba t de Student para el análisis del número total de respuestas correctas (aciertos) y los promedios de tiempo de respuesta de cada estímulo en las dos tareas del paradigma; se obtuvieron

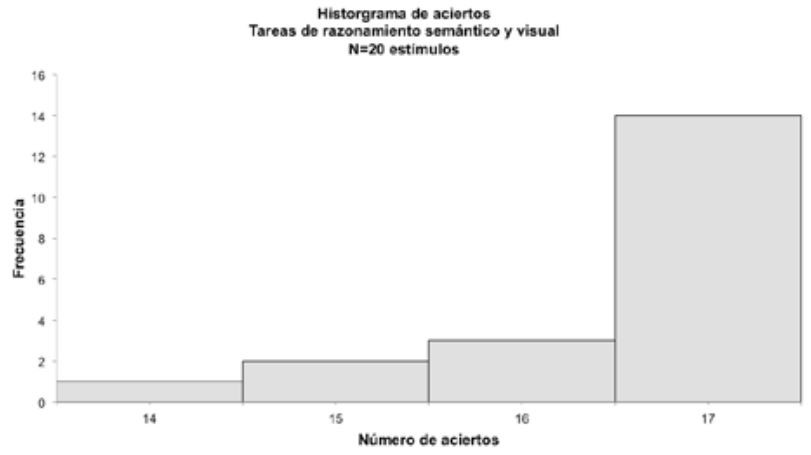

Figura 5. Histograma de frecuencia de aciertos de los 17 participantes en los 20 estímulos del paradigma.

valores críticos a 16 grados de libertad (2.120) con un nivel de confianza de $95 \%$.

Luego, se compararon los valores normalizados de t por estímulo y se rechazaron aquellos con valores superiores al nivel de confianza, mientras que se aceptaron aquellos dentro del nivel de confianza e inferior a éste. Para ese análisis, el número de respuestas correctas se tomó como un indicador de "comprensión" del ejercicio, mientas que el tiempo de ejecución se tomó como un indicador de "dificultad" de la tarea.

La Tabla 3 muestra los resultados obtenidos en la tarea de razonamiento semántico, donde el promedio de respuestas correctas fue de 16 aciertos, mientras que el tiempo promedio de respuesta fue de $2.3 \mathrm{~s}$ por estímulo. En esta tarea se rechazaron 2 estímulos, el par reloj-regla y

Tabla 3. Número de respuestas correctas y el tiempo promedio de respuesta de los 17 sujetos por cada estímulo de la tarea de razonamiento semántico.

\begin{tabular}{|c|c|c|c|c|c|c|c|}
\hline \multicolumn{8}{|c|}{ Tarea de razonamiento semántico } \\
\hline \multicolumn{4}{|c|}{ Análisis de comprensión ( $N=17$ ) } & \multicolumn{4}{|c|}{ Análisis de dificultad ( $N=17)$} \\
\hline $\begin{array}{l}\text { Estímulo } \\
\text { semántico }\end{array}$ & $\begin{array}{c}\text { Total respuestas } \\
\text { correctas }\end{array}$ & $\stackrel{t}{\text { normalizado }}$ & Resultado & Total tiempo & $\begin{array}{l}\text { Tiempo promedio } \\
\text { respuesta }\end{array}$ & $\stackrel{\mathbf{t}}{\text { normalizado }}$ & Resultado \\
\hline 1. tren-bicicleta & 17 & 1.00 & Acepto & $39.9 \mathrm{~s}$ & $2.3 \mathrm{~s}$ & -0.28 & Acepto \\
\hline 2. reloj-regla & 17 & 1.00 & Acepto & $49.7 \mathrm{~s}$ & $2.9 \mathrm{~s}$ & 3.24 & Rechazo \\
\hline 3. ojo-nariz & 17 & 1.00 & Acepto & $32.7 \mathrm{~s}$ & $1.9 \mathrm{~s}$ & -2.83 & Acepto \\
\hline 4. perro-caballo & 17 & 1.00 & Acepto & $31.1 \mathrm{~s}$ & $1.8 \mathrm{~s}$ & -3.41 & Acepto \\
\hline 5. codo-rodilla & 15 & -9.00 & Rechazo & $58.8 \mathrm{~s}$ & $3.4 \mathrm{~s}$ & 6.49 & Rechazo \\
\hline 6. camisa-zapato & 17 & 1.00 & Acepto & $36.2 \mathrm{~s}$ & $2.1 \mathrm{~s}$ & -1.59 & Acepto \\
\hline 7. invierno-verano & 17 & 1.00 & Acepto & $30.3 \mathrm{~s}$ & $1.7 \mathrm{~s}$ & -3.68 & Acepto \\
\hline 8. mariposa-abeja & 17 & 1.00 & Acepto & $43.4 \mathrm{~s}$ & $2.5 \mathrm{~s}$ & 0.99 & Acepto \\
\hline 9. madera-ladrillos & 17 & 1.00 & Acepto & $42.6 \mathrm{~s}$ & $2.5 \mathrm{~s}$ & 0.71 & Acepto \\
\hline 10.leche-agua & 17 & 1.00 & Acepto & $41.6 \mathrm{~s}$ & $2.4 \mathrm{~s}$ & 0.36 & Acepto \\
\hline \multicolumn{4}{|c|}{ Media $=16.8$ Desviación Estándar $=0.63$} & \multicolumn{4}{|c|}{ Media $=2.3 \mathrm{~s} \quad$ Desviación Estándar $=0.52 \mathrm{~s}$} \\
\hline
\end{tabular}


Tabla 4. Número de respuestas correctas y el tiempo promedio de respuestas de los 17 sujetos por cada estímulo de la tarea de razonamiento visual.

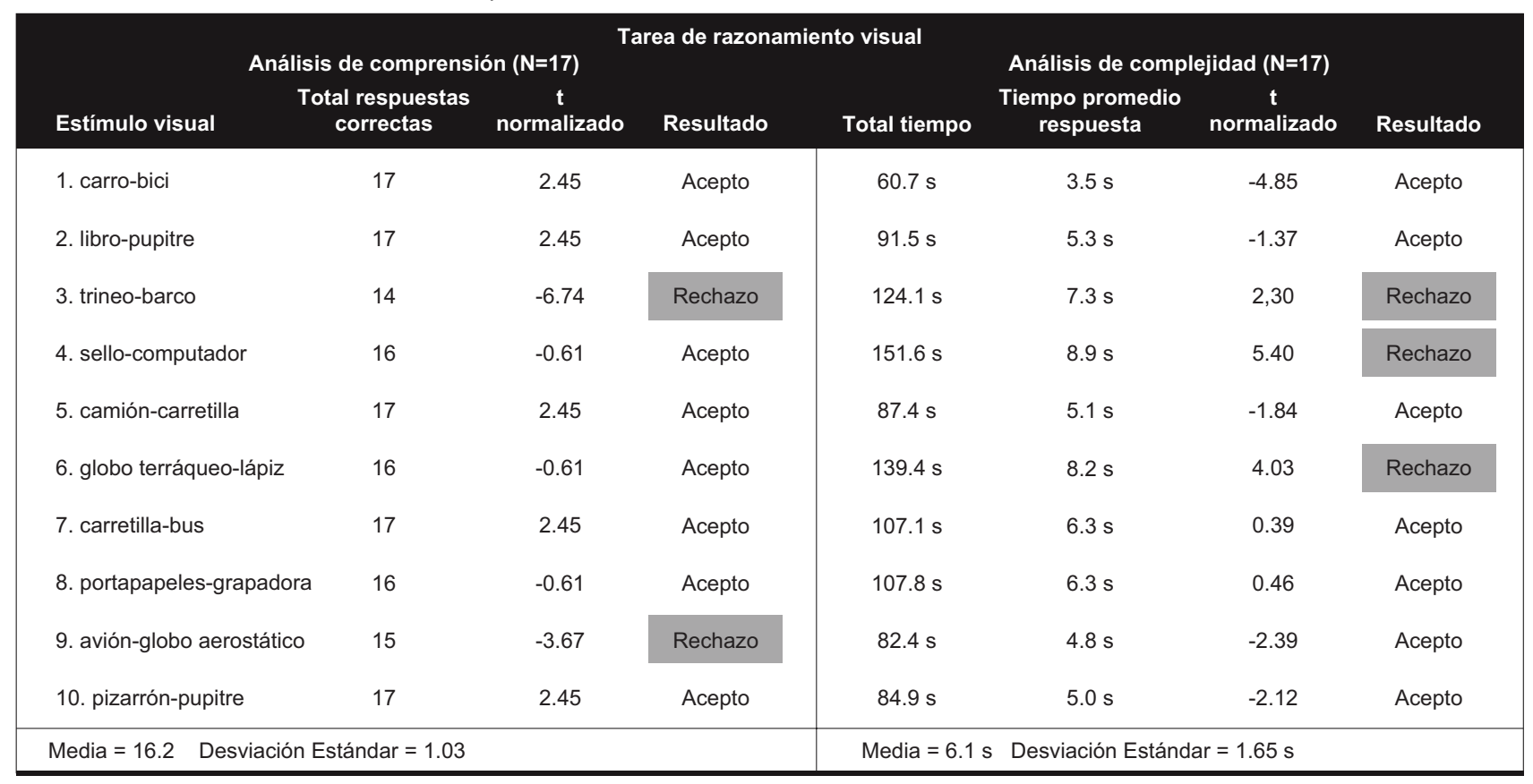

el par codo-rodilla debido a problemas de comprensión y dificultad para resolverlos.

La Tabla 4 muestra que el promedio de respuestas correctas en la tarea de razonamiento visual fue de 16 aciertos, mientras que el tiempo promedio de respuesta fue de $6.1 \mathrm{~s}$ por estímulo. En esta tarea se rechazaron 4 estímulos por problemas de comprensión y grado de dificultad.

Los datos anteriores aportan evidencia en contra de la hipótesis nula (Ho) y a favor de la hipótesis de investigación (Hi). Así, el tiempo de emisión de respuesta en la tarea de razonamiento visual fue mayor al tiempo de emisión de respuesta de la tarea de razonamiento semántico.

\section{Discusión}

Para Maestú et al.$^{18}$ un paradigma debe estar validado en sujetos sanos antes de su aplicación dentro de un RMf. Con ello se pretende que la tarea experimental active las áreas cerebrales específicas relacionadas con la actividad cognitiva propuesta. En el razonamiento abstracto la red neurológica involucrada en la resolución de paradigmas analógicos está formada por un sistema frontoparietal-bilateral en asociación con la corteza cingulada anterior y regiones temporales?

Como muestra la Figura 4, se eliminó a un sujeto del estudio debido a que sus tiempos de respuesta fueron estadísticamente atípicos. Por ejemplo, en el estímulo 5 de razonamiento semántico, el participante respondió en 10.2s, cuando el promedio de respuesta en ese reactivo fue de 3.8s. Lo mismo sucedió en el estímulo 6 de razonamiento visual donde respondió a los $22.8 \mathrm{~s}$, mientras que el promedio en dicho ítem fue de $9 \mathrm{~s}$. Un patrón similar de res- puesta se presentó en los demás estímulos, por lo que el participante fue eliminado de la muestra final.

En dicho sujeto, posiblemente, se presentó cierta confusión, sin embargo, para la mayoría de participantes el paradigma fue comprensible. En ese sentido, la Figura 5 muestra que las respuestas correctas de todo el estudio se agruparon en el $85 \%$ de los participantes, lo que indica que el experimento fue resoluble. No obstante, el análisis estadístico del paradigma permitió identificar y rechazar aquellos reactivos en los que los sujetos cometieron más errores o superaron el tiempo promedio de ejecución.

Así, de los veinte reactivos que formaron parte del estudio, sólo dos de ellos fueron rechazados estadísticamente tanto en el análisis de comprensión, como en el análisis de dificultad. Ese fue el caso de los estímulos 5 (codo-rodilla) de la tarea de razonamiento semántico y el estímulo 3 (imágenes trineo y barco) de la tarea de razonamiento visual. En el reactivo 5 la complejidad quizás se debió a la similitud de las opciones de respuesta (flexionan-conectan), mientras que en el reactivo 3 , es probable que la complejidad se presentó en el reconocimiento visual de la imagen del trineo, puesto que es un objeto poco familiar en nuestro medio.

También se rechazaron cuatro estímulos que no pasaron sólo uno de los análisis. Por ejemplo, el reactivo 2 (reloj-regla) de la tarea de razonamiento semántico fue aceptado en el análisis de comprensión, pero rechazado en el análisis de complejidad ya que su tiempo promedio de resolución fue superior al nivel de confiabilidad. Lo mismo sucedió con los estímulos 4 (imágenes sello-computador), 6 (imágenes globo terráqueo-lápiz) y 9 (imágenes aviónglobo aéreo estático) de la tarea de razonamiento visual. 
Eso significa que en algunos casos los reactivos fueron fáciles de comprender, pero difíciles de resolver. Es posible que los problemas que presentaron los estímulos visuales rechazados se deban a la dificultad para identificar de manera rápida y sencilla la imagen empleada.

Por otra parte, el estudio arrojó datos a favor de la hipótesis de investigación evidenciando que el tiempo para contestar los reactivos de la tarea de razonamiento semántico (media de $2.3 \mathrm{~s}$ ) fue mucho menor al tiempo empleado en la resolución de los estímulos de la tarea de razonamiento visual (media de $6.1 \mathrm{~s}$ ). Esa diferencia en los tiempos de respuesta se podría explicar por la cantidad de procesamiento cognitivo invertido, más que por el grado de dificultad de los estímulos. En la tarea semántica sólo se leen las palabras diana, se establece la relación abstracta y se elige una respuesta. En cambio, en la tarea visual, se deben reconocer las imágenes diana, identificar el número que le corresponde a cada una de ellas, se establece la relación abstracta y se busca la respuesta entre dos opciones. Es decir, se realizan alrededor de cuatro procesos visuales y un proceso cognitivo.

Esas diferencias de tiempo de exposición entre estímulos escritos e imágenes también se observaron en los estudios de Whitaker et al. ${ }^{10}$ quienes usaron imágenes a color con un tiempo de exposición de $10 \mathrm{~s}$ y Hammer et al..$^{12}$ que emplearon estímulos gráficos acompañados de palabras durante $8 \mathrm{~s}$. Mientras que Modi et al. ${ }^{11}$ sólo mostraron durante $4 \mathrm{~s}$ figuras geométricas simples. Al parecer, los estímulos que usan gráficos complejos necesitan más tiempo de exposición que los semánticos.

Nuestros hallazgos sobre el tiempo de exposición de estímulos indican que para mapear la actividad cerebral durante cuatro minutos en un equipo de RM de 1.5T, como sugiere Aguirre-Reyes, ${ }^{17}$ la tarea de razonamiento semántico debe de contar con el doble de estímulos planteados en este estudio, es decir, se necesitan aproximadamente 24 reactivos con un tiempo de exposición de $2.5 \mathrm{~s}$ cada uno. Mientras que para la tarea de razonamiento visual el número de reactivos a emplearse queda establecido en 10 estímulos con un tiempo de exposición de $6 \mathrm{~s}$ cada uno.

De esa manera, el paradigma de razonamiento abstracto quedaría conformado por 8 bloques ( 4 de actividad y 4 de reposo) de $30 \mathrm{~s}$ cada uno, con lo que se tendría un tiempo total de cuatro minutos de mapeo cerebral. Con ello se aumentaría la potencia de contraste y significancia estadística cuando se emplee el paradigma en una tarea de $\mathrm{RMf}^{14,15}$

La validación de nuestro paradigma permitió identificar aspectos importantes a considerar en su diseño final, los cuales evitarían que el mapeo del cerebro en un RMf se dé entorno a la confusión o a los errores de resolución de los ejercicios, aumentando la probabilidad de que la actividad cerebral registrada provenga de la solución comprensible de la tarea experimental. Así, la validación del paradigma en relación al número de aciertos hallados y el tiempo promedio de ejecución documentado, garantiza con un $95 \%$ de confiabilidad que las personas serán capaces de entenderlo y resolverlo.

Respecto a las limitaciones prácticas de la investigación se tiene que la escasez de literatura relacionada con RM y la validación de paradigmas para funciones cognitivas superiores dificultó el diseño metodológico de la tarea experimental. Los tiempos de actividad y reposo, así como el número de estímulos de cada tarea se basaron en las generalidades de un diseño de bloques y en el asesoramiento de un grupo de expertos. Eso se evidenció en la selección de los gráficos y de las opciones de respuestas que, en algunos casos, fueron confusas y complicaron el experimento. Si se realiza las modificaciones de ciertos estímulos visuales y semánticos se obtendrán mejores resultados y se contará con un mayor número de bloques de actividad.

Por otra parte, a pesar de la ventaja que implica el acceso libre del PsychoPy para la investigación en las neurociencias, su uso requiere de una vasta experiencia en programación informática. Y aunque no es complicado familiarizarse con el entorno básico y amigable del programa, en contraste con el uso del PEBL, ${ }^{19}$ fue necesario la colaboración de un ingeniero en computación, lo que implicó tiempo para la capacitación y programación del paradigma.

Si bien este estudio es pionero en la propuesta de validar un paradigma de razonamiento abstracto, aún estamos lejos de obtener una versión válida y confiable. En futuras investigaciones se deben tomar en cuenta nuestros hallazgos con el objetivo de generar un paradigma final que, posteriormente, deberá ser probado con RMf de manera práctica. Sólo hasta entonces se podrá contar con un instrumento confiable que pueda ser validado con otras técnicas de neuroimagen.

\section{Conclusiones}

El presente estudio validó un paradigma de razonamiento abstracto para RMf a través del análisis estadístico de comprensión y complejidad de la tarea. Se concluyó que el paradigma necesita más del doble de estímulos para la tarea de razonamiento semántico, dado que su tiempo de resolución fue significativamente menor al tiempo de ejecución de la tarea de razonamiento visual, por lo que es necesario incluir más reactivos semánticos y corregir los estímulos visuales rechazados que deberán pasar por un nuevo proceso de validación previo a su uso en una tarea de RMf.

Finalmente, la investigación aportó nuevos conocimientos al incipiente campo de investigación neuropsicológica del Ecuador. Estos hallazgos proporcionaron una propuesta metodológica para la creación y validación de nuevas tareas experimentales para técnicas de neuroimagen. Con ello, también se potencia el uso de la neuroimagen como una herramienta de investigación científica y no 
sólo como un medio de diagnóstico neurológico. Estos resultados documentaron el número necesario de estímulos y su tiempo promedio de exposición a considerase en el diseño de un paradigma de razonamiento abstracto para un RM. Todavía hay mucho que contribuir a la validación de este paradigma, por lo que se abre un amplio campo para futuras investigaciones desde el área de la neuropsicología.

\section{Referencias}

1. Armony J, Trejo D, Hernández D. Resonancia Magnética Funcional (RMf): principios y aplicaciones en Neuropsicología y Neurociencias Cognitivas. Neuropsicología Latinoamericana. 2012; 4(2): 36-50.

2. Aguirre-Reyes D, Bustamante B. Mapeo de la Función Cerebral. BIOscientis Revista de Divulgación Científica. 2014; 1(2): 44-48.

3. Esparza N. Mapeo de la función cerebral en adultos mayores enfocados en la tarea de Funciones Ejecutivas. Loja: Universidad Particular de Loja; 2017.

4. Llivisaca L. Mapeo de la función cerebral en adultos mayores enfocado en la tarea de atención. Loja: Universidad Particular de Loja; 2017.

5. Palacios B. Mapeo de la función cerebral en asultos mayores enfocado en la tarea de Lenguaje. Loja: Universidad Particular de Loja; 2017.

6. Gutiérrez M. Diseño e implementción de una herramienta computacional dinámica para mejorar las habilidades de memoria de adultos mayores, y comprobar su eficacia por medio de la detección de actividad cerebral utiliando imágenes de resonancia magnética. Loja: Universidad Particular de Loja; 2017.

7. Valadez J, Granados E. Neuroanatomía funcional para neuropsicólogos. México DF: Universidad Nacional Autónoma de México; 2012.

8. Jaramillo L, Puga L. El pensamiento lógico-abstracto como sustento para potenciar los procesos cognitvios en la educación. Sophia, colección de Filosofía de la Educación. 2016; 21(2): 31-55.

9. Hobeika L, Diard-Detoeuf C, Garcin B, Levy R, Volle E. General and Specialized Brain Correlates for Analogical Reasoning: A Meta-anlysis of Functional Imaging Studies. Human Brain Mapping. 2016; 37(5): 1953-1969.

10. Whitaker K, Vendetti M, Wendelken C, Bunge S. Neuroscientific insights into the development of analogical reasoning. Developmental Science. 2017; 21(2): 1-11.

11. Modi S, Kumar M, Nara S, Kumar P, Khushu S. Trait anxiety and neural efficiency of abstract reasoning: An fMRI investigation. Journal of Biosciences. 2018; 43(5): 877-886.
12. Hammer R, Paul E, Hillman C, Kramer A, Cohen N, Barbey A. Individual differences in analogical reasoning revealed by multivariante task-based functional brain imagin. NeuroImagen. 2019; 184: 993-1004.

13. Ramos C, Ramos V, Jadán J, Lepe N, Paredes L, Gómez A, Bolaños M. Conceptos fundamentales en la teoría neuropiscológica. Revista Ecuatoriana de Neurología. 2017; 26(1): 53-60.

14. Ríos-Lago M. Neuropsicología y resonancia magnética funcional: Conceptos generales. Radiología. 2008; 50(5): 351-365.

15. Manes F, Báez S. Métodos de investigación en Neurociencias Cognitivas. En: Labos E, Slachevsky A, Torralva T, Fuentes P, Manes F, Editores. Tratado de Neuropsicología Clínica. 2 ed. Buenos Aires: Akadia. 2019. p.69-104.

16. Cortés P, Gutiérrez C, Jara C, Peña M. Neuroimagen y Cognición. En Labos E, Slachevsky A, Torralva T, Fuentes P, Manes F, Editores. Tratado de Neuropsicología Clínica. 2 ed. Buenos Aires: Akadia. 2019. p. 35-49.

17. Aguirre-Reyes D. Tamaño de muestra a considerarse en un estudio de Resonancia Magnética funcional (RMf) con un equipo de resonancia magnética de 1.5. T. Revista Ecuatoriana de Neurología. 2012; 21(1-3): 10-14.

18. Maestú F, Ríos-Lago M. Aplicaciones clínicas de la RM funcional y la MEG. En Pérez M, Coordinador. Manual de Neuropsicología clínica. Madrid: Pirámide. 2009.p. 67-91.

19. Ramos C, Jadán J, Ramos D, Bolaños M, Ramos V, Fiallo, M. Evaluación Neuropsicológica del Control Inhibitorio y el Control de la Interferencia: Validación de Tareas Experimentales en el Contexto Ecuatoriano. Revista Ecuatoriana de Neurología. 2017; 26 (1): 27-34.

20. Hernández R, Fernández C, Baptista P. Metodología de la investigación. México DF: McGraw-Hill; 2014.

21. Peirce J. Generating stimuli for neuroscience using PsychoPy. Frontiers in Neuroinformatics. 2009; 2 (10): $1-8$.

22. Wechsler D. Escala de inteligencia para niños-IV: manual de aplicación. México DF: Manual Moderno; 2007.

23. Simon J, Rudell A. Auditory SR compatibility: the effect of an irrelevant cue on information processing. Journal of Applied Psychology. 1967; 51(3): 300-304.

24. Simon J. Reactions toward the source of stimulation. Journal of experimental Psychology. 1969; 81 (1): $174-176$. 